\title{
Recent Advances in the Use of Drosophila melanogaster as a Model to Study Immunopathogenesis of Medically Important Filamentous Fungi
}

\author{
Georgios Hamilos, ${ }^{1}$ George Samonis, ${ }^{1}$ and Dimitrios P. Kontoyiannis ${ }^{2}$ \\ ${ }^{1}$ Department of Internal Medicine, School of Medicine, University of Crete, Stavrakia, Voutes, \\ 71110 Heraklion, Crete, Greece \\ ${ }^{2}$ Department of Infectious Diseases, Infection Control and Employee Health, The University of \\ Texas MD Anderson Cancer Center, 1515 Holcombe Boulevard, Houston, TX 77030, USA \\ Correspondence should be addressed to Dimitrios P. Kontoyiannis, dkontoyi@mdanderson.org
}

Received 18 August 2011; Accepted 7 November 2011

Academic Editor: Nir Osherov

Copyright ( $) 2012$ Georgios Hamilos et al. This is an open access article distributed under the Creative Commons Attribution License, which permits unrestricted use, distribution, and reproduction in any medium, provided the original work is properly cited.

\begin{abstract}
Airborne opportunistic fungi, including Aspergillus and other less common saprophytic molds, have recently emerged as important causes of mortality in immunocompromised individuals. Understanding the molecular mechanisms of host-fungal interplay in robust experimental pathosystems is becoming a research priority for development of novel therapeutics to combat these devastating infections. Over the past decade, invertebrate hosts with evolutionarily conserved innate immune signaling pathways and powerful genetics, such as Drosophila melanogaster, have been employed as a means to overcome logistic restrains associated with the use mammalian models of fungal infections. Recent studies in Drosophila models of filamentous fungi demonstrated that several genes implicated in fungal virulence in mammals also play a similarly important pathogenic role in fruit flies, and important host-related aspects in fungal pathogenesis are evolutionarily conserved. In view of recent advances in Drosophila genetics, fruit flies will become an invaluable surrogate model to study immunopathogenesis of fungal diseases.
\end{abstract}

\section{Introduction}

In recent years, opportunistic fungi have emerged as leading causes of morbidity and mortality in immunocompromised individuals [1-3]. Aspergillus is by far the most common of these molds, and mortality rates for invasive aspergillosis exceed $90 \%$ in hematopoietic stem cell transplant recipients $[4,5]$. Even more concerning, however, is that infections caused by other difficult-to-treat opportunistic molds, such as Mucorales species, are increasingly being observed in several cancer centers [6-8]. The increase in the frequency and spectrum of invasive fungal infections in immunocompromised patients underscores the need for expanding our knowledge of the pathogenesis of opportunistic fungal infections and developing novel therapeutic approaches.

The versatility and complexity of virulence mechanisms and predisposing host conditions that lead to development of invasive mold infections $[9,10]$ necessitate understanding the nature of host-fungal interactions at the cellular and molecular levels in order to identify host immune pathways and pathogen determinants involved in disease progression $[11,12]$. Pioneering studies over the past decade demonstrated that a variety of opportunistic fungi can invade and cause fatal infection in a variety of simple invertebrate hosts, such as the fruit fly Drosophila melanogaster, and the roundworm Caenorhabditis elegans [13-20]. Also, it has become evident from these studies that important aspects of innate immunity have been evolutionarily conserved across phylogeny. Thus, because of their simplicity, well characterized innate immune signaling pathways, and because both the host and pathogen are amenable to genetic analysis and high-throughput screening in each of these pathosystems, the use of invertebrate models has accelerated studies of microbial virulence and host immunity [21-24]. In addition, 
because of their low cost, small size, and short generation time, invertebrate hosts have been used in mass screening assays for selection of antimicrobial compounds with novel mechanisms of action. In this review, we outline recent advances in the study of medically important filamentous fungi in Drosophila model and discuss future implications and challenges in the use of this elegant pathosystem.

\section{Antifungal Innate Immune Pathways in Drosophila melanogaster}

2.1. Humoral Antifungal Immune Responses. Although lacking adaptive immunity, invertebrates are capable of having efficient innate immune responses against an array of pathogens in their natural environments. Two major pathways orchestrate innate immune responses in $D$. melanogaster, the immune deficiency (imd) pathway that confers protection against gram-negative bacteria, and the Toll pathway that is critical for immunity against grampositive bacteria and fungi [25]. Detection of invading microorganisms by host receptors of the peptidoglycan recognition protein (PGRP) or gram-negative binding protein (GNBP) families triggers the activation of signal transduction pathways in the fat body (liver analogue) via the Toll receptor leading to a systemic humoral response characterized primarily by massive synthesis and release of potent antimicrobial peptides. Despite the broad spectrum of antimicrobial peptides, some specificity exists upon their induction following infection by various microbial pathogens. For example, in D. melanogaster, fungi and grampositive bacteria mainly induce the production and release of drosomycin and metchnikowin via the Toll pathway, whereas gram-negative microbes induce the production and release of diptericin, attacin, and cecropin via the imd pathway [25]. The predominant role of the Toll pathway in Drosophila immunity against Aspergillus was first demonstrated in a landmark study by Lemaitre et al. [17], who found that Toll mutant flies, in contrast to wild-type flies, were highly susceptible to Aspergillus infection.

In both insects and mammals, the interaction of immunostimulatory cell wall molecules of invading fungi with Toll receptor(s) leads to activation of intracellular phosphorylation cascades, with subsequent translocation of nuclear factor $\kappa \mathrm{B}$-like transcriptional factors to the nucleus and induction of antimicrobial peptide-encoding genes [10, 25]. However, in contrast to mammalian Toll receptors, there is no direct interaction between microbial ligands and Drosophila Toll receptor. Instead, activation of the Toll signaling cascade is mediated by GNBP-3, a soluble pattern recognition receptor that senses long-chain fungal b-(1-3)glucans and triggers a serine protease cascade leading to the processing of a small cytokine-like molecule, Spätzle, which comprises the functional ligand of the Toll receptor [25]. Importantly, GNBP3 mutant flies are highly susceptible to opportunistic fungi, including Candida and Aspergillus while retain functional Toll pathway activity, implying Tollindependent immune-related functions of this receptor. Indeed, GNBP3 has been implicated to play a role in pathogen agglutination, and activation of the melanization reaction at the early stages of fungal invasion [26]. Importantly, parallel to GNBP3, a second detection system senses the activity of proteolytic virulence factors that are released in the fly hemolymph during invasive fungal growth and redundantly activates Toll pathway via the protease Persephone [27].

2.2. Cellular Antifungal Immune Responses. When compared to humoral immune responses, D. melanogaster cellular immune responses are less well characterized. Notably, recent studies in insects challenge the importance of humoral immunity in pathogen clearance, demonstrating that the vast majority of bacteria (99.5\%) are rapidly eliminated from the haemolymph well before the induction of antimicrobial peptides [28]. Hence, cellular immune responses seem to play instrumental roles in early recognition and elimination of microbial pathogens. The key transcription factor downstream of the Toll pathway, the nuclear factor- $\kappa \mathrm{B}$ homologue Dif, is required for regulation of both humoral and cellular immunity in flies [25]. Phagocytosis is a hallmark of the cellular immune response and exhibits considerable similarity across phylogeny. Hence, opsonization and recognition by specific receptors mediate the initial stages of phagocytosis in both invertebrates and mammals. For example, in Drosophila peptidoglycan, recognition proteins (PGRPs) such as PGRPLC and Croquemort (a human CD36 homologue) participate in the recognition and phagocytosis of gram-negative bacteria $[29,30]$, whereas the transmembrane scavenger receptor eater has been shown to recognize bacteria and fungi (Candida silvata) and play a Toll-independent role in antifungal immunity [23, 31]. Of interest, thioestercontaining proteins with a complement-like activity against invading pathogens have been identified in many insects, including fruit flies $[25,32]$. A high-throughput screen in Drosophila melanogaster S2 RNAi library identified a novel protein, macroglobulin complement related $(\mathrm{Mcr})$, that exerts opsonizing activity specifically against Candida albicans [33]. In addition, S2 Drosophila cells efficiently eliminate C. albicans yeast cells and induce significant damage to the hyphae of filamentous fungi, including Aspergillus and the Mucorales, in a way that resembles the antifungal effector function of human phagocytes $[23,34]$.

The molecular mechanisms of intracellular elimination of pathogens by Drosophila phagocytic cells are less well characterized. Thus, insect phagocytic cells are also capable of generating an oxidative burst of oxygen radical intermediates, whereas induction of nitric oxide synthase has been shown to protect against bacterial infection in Drosophila larvae [35]. Furthermore, numerous antimicrobial peptides contained within human neutrophil granules, such as lysozyme, lipases, metalloproteases (like the mammalian gelatinases or collagenases), and nucleases, are similarly produced by the phagocytic hemocytes of most insects in response to infection $[11,25]$. Although little is known about the molecular mechanisms of intracellular elimination of pathogens in fruit flies, recent studies demonstrate that the evolutionarily conserved autophagy pathway is important 
for immune surveillance and clearance of intracellular pathogens that escape into the cytoplasm, including Cryptococcus [36]. On the other hand, unique cellular responses against larger invading pathogens (e.g., parasites), such as encapsulation and melanization mediated by specialized immune effector cells, are seen in Drosophila and other insects $[11,25]$.

2.3. Epithelial Immune Responses. In D. melanogaster, antimicrobial peptide-encoding genes are constitutively expressed in epithelia that are in direct contact with the external environment. However, in contrast with the systemic immune responses mediated by the fat body, where the Toll pathway modulates immune responses against grampositive bacteria and fungi, epithelial immune responses in D. melanogaster appear to be partially controlled by the Imd pathway [25]. Furthermore, recent evidence indicates that genes involved in oxidative stress and/or detoxification of reactive oxygen species are critical for epithelial defense [37]. In addition, recent studies demonstrated a major role for the Janus kinase- (JAK-) signal transducer and activator of transcription (STAT) signaling pathway in epithelial host defense via regulation of stem cell proliferation and epithelial cell homeostasis [38].

A recent study in a gastrointestinal infection (GI) model of candidiasis in Drosophila larvae demonstrated an important role of normal gut flora in epithelial immunity by preventing colonization and invasive infection by Candida, which resembles the increasingly appreciated regulatory role of human epithelial microbiota in shaping epithelial immune responses [39]. Of interest, activation of JNK signaling during Candida infection accounted for extensive epithelial cell death in the gut and mortality of Drosophila larvae. In parallel, Candida infection triggered a systemic protective immune response that was mediated by NO release from larvae hemocytes and the parallel activation of the Toll pathway by pathogen-secreted aspartyl proteinases.

\subsection{Toll-Independent Innate Immune Pathways in Drosophila.} The complexity of the immune defenses in insects is much higher than initially perceived, and cross-talk between the Imd and Toll pathways takes place in response to both gramnegative and gram-positive microbes [25]. Furthermore, besides the Toll and Imd signaling cascades, other pathways associated with developmental or stress resistance processes are induced in response to infections in both invertebrates and mammals. For example, a pioneer study in Drosophila demonstrated that antimicrobial peptide activation can be achieved independently of classic immunoregulatory pathways by the transcription factor FOXO, a key regulator of stress resistance, metabolism, and aging [40]. In uninfected animals, antimicrobial peptide genes are activated in response to nuclear FOXO activity when induced by starvation or by using insulin-signaling mutants, revealing a new mechanism of cross-regulation of metabolism and innate immunity that has proven to be functional in humans as well [40]. Furthermore, investigators showed that the activation of the evolutionarily conserved p38 MAPK pathway is important for resistance to infection by bacteria and fungi; of interest, in contrast to the mammalian homologue, activation of p38 MAPK occurs independently of the Toll signaling [41].

\section{Modeling Microbial Infection in Drosophila melanogaster}

In D. melanogaster, the pathogen of interest is typically injected into the dorsal thorax via either needle pricking or microinjection [11]. In regard to fungal pathogens, the injection assay is technically a more standardized and reproducible method of infection and allows for a more precise estimation of fungal inoculums. Nonetheless, parenteral inoculation by passes the physiologic route of entry of the pathogen of interest and results in a more overwhelming infection that may not be suitable for pathogenesis studies. Thus, other more physiologic methods of infection are also used. For example, the alb1 Aspergillus fumigatus mutant, which is hypovirulent in mice, exhibited attenuated virulence in Toll-deficient flies only when introduced by feeding or rolling [20]. These infection methods are typically achieved by feeding insects in a lawn of yeast or molds or rolling insects over a fresh carpet of fungal spores. However, standardization of the infecting inocula is difficult with natural infection methods such as ingestion. Furthermore, infection with molds other than Aspergillus by feeding and rolling is difficult to perform because of the distinct pattern of growth of fungal colonies.

Female flies are typically used in infection experiments because of their larger size and relative resistance to injection injury when compared with male flies. Because wild-type Drosophila is resistant to most pathogenic fungi and bacteria, mutants deficient in various components of the Toll cascade are frequently employed to model infections. In most cases, crossing different loss-of-function alleles is required to generate homozygous Toll-mutant flies [11]. Nonetheless, the need for crossing of fly strains is a limitation for high throughput screening assays. Of note, microinjection introduces significantly higher inoculums within Drosophila hemolymph than needle pricking that allowed for establishment of invasive Candida infection in wild-type Drosophila melanogaster flies [42].

A major advantage of Drosophila in comparison to all other model host organisms is its genetic tractability, wellcharacterized immune system, and remarkable degree in conservation of biochemical pathways that control fundamental physiologic processes such as cell proliferation, differentiation, and tissue homeostasis. Furthermore, the innate susceptibility of Drosophila Toll mutant strains to fungal infections obviates the need to use immunosuppressive agents, thus eliminating the host variability inherent in the use of immunosuppressive regimens. In particular, Drosophila strains are amenable to both forward and reverse genetics, and large collections of Drosophila mutants and transgenic cell lines are commercially available (http://flybase.net/). Also, the Drosophila genome sequence 
was one of the first to be completed and is probably one of the most fully annotated eukaryotic genomes found in a database (http://flybase.net/annot/). As a result, double-stranded RNA has been synthesized for each of the Drosophila genes (http://www.flyrnai.org/) and recently lines expressing RNAi have become available, which allow for conditional inactivation of every single gene at a whole animal or tissue level (http://www.vdrc.at/).

\section{Virulence Studies of Filamentous Fungi in Drosophila melanogaster}

4.1. Aspergillus. Since filamentous fungi have been in existence for about 1 billion years, the fly immune system evolved in the face of continued exposure to airborne conidia. Thus, Drosophila immune system has developed highly sophisticated and efficient strategies to combat infection caused by Aspergillus and other filamentous fungi. In fact, only a few entomopathogenic fungi are able to infect fruit flies in nature, via penetration of fly exoskeleton. Even when fungal pathogens are experimentally introduced directly into the fly hemolymph, wild-type flies are still capable of effectively eliminating infection. Lemaitre and colleagues were the first to demonstrate that Aspergillus fumigatus was able to infect and kill flies carrying mutations in various aspects of the Toll pathway [17]. Toll-deficient flies have been since implemented as a model to study immunopathogenesis of infections caused by Aspergillus and other medically important filamentous fungi. Several virulence attributes of Aspergillus pathogenicity in mammals have been tested in the fly model $[20,43]$. With the exception of virulent factors that are important for microbial survival at mammalian temperature [44], most other virulence attributes that are important for mammalian pathogenicity of Aspergillus were equally important for successful infection in Toll-deficient fruit flies. In particular, Aspergillus mutants that are defective in siderophore biosynthesis (DeltasidA, DeltasidD), PABA metabolism (H515), starvation stress response, secondary metabolite production (DgliP), or melanin biosynthesis were attenuated in both Drosophila and mouse models of invasive aspergillosis [20,43]. Notably, fungal cell wall melanin was dispensable for Aspergillus virulence when fungal spores were injected into the fly hemolymph but was important for establishment of invasive infection though Drosophila epithelia [20]. Hence, the tempo and site of infection as well as differences in local host defense mechanisms may influence expression of virulence factors of fungi in the fly model. Evermore, similar to recent findings with the $\Delta C g r A$ mutant [44], putative virulent factors of $A$. fumigatus with a role in thermotolerance may not be encountered in Drosophila or other invertebrate models because infection in these minihosts takes place at temperatures much lower $\left(25^{\circ} \mathrm{C}\right)$ than the mammalian physiologic temperature $\left(37^{\circ} \mathrm{C}\right)$. Despite these limitations, accumulating experimental evidence suggests that Drosophila is a relevance model to study Aspergillus virulence.

The interstrain and interspecies variations in virulence for a collection of Aspergillus fumigatus and Aspergillus terreus clinical isolates were recently studied in Toll-deficient fruit flies [45]. Although there was no significant difference in the survival of flies infected with $A$. fumigatus versus $A$. terreus or flies infected with colonizing versus invasive isolates of either species, two dominant A. fumigatus clades identified by rep-PCR were associated with significantly different survival rates in Toll-deficient flies. Therefore, the fly model of aspergillosis could detect subtle changes in virulence and uncover distinct $A$. fumigatus clades that differ in their pathogenicity. Of interest, a similar pathogenicity study of Candida albicans clinical isolates that were previously ranked for virulence in mice was recently performed in wild-type Drosophila flies infected by microinjection [42]. Of interest, there was a significant correlation in virulence of $C$. albicans strains between the fly and the mouse model of disseminated candidiasis. Nonetheless, differences in virulence were not evident using immune-deficient spatzle ${ }^{-/-}$flies, suggesting that Toll signalling might actually be required to predictably differentiate virulence.

The recent completion of the sequencing of the A. fumigatus genome and the development of molecular toolsets to study the biology of A. fumigatus is expected to lead to the generation of multiple Aspergillus mutants and creates a need for high-throughput strategies capable of assessing the contribution of individual genes to Aspergillus virulence [46]. Validation of Drosophila as a suitable model for largescale virulence studies was provided by a recent screen of 34 Candida albicans mutants defective in putative transcription factor genes. This study identified a novel transcriptional regulator of cell wall integrity, CAS5, which proved to be important for virulence in both Drosophila and the mouse model of invasive candidiasis; a parallel screen in $C$. elegans subsequently confirmed the role of CAS5 in Candida virulence [47].

4.2. Mucorales (Formerly Zygomycetes). Mucorales species have recently emerged as an important cause of serious angioinvasive infections in immunocompromised individuals [6-8]. Rhizopus species accounts for majority of cases of mucormycosis in humans [7]. Few animal models of mucormycosis exist, and the immunopathogenesis of this infection is largely unknown. However, sequencing of Rhizopus oryzae genome has been completed and genetic tools are available (http://www.broad.mit.edu/annotation/ fungi/rhizopus_oryzae/). In immunocompetent individuals, blood and tissue phagocytes efficiently eliminate Mucorales spores and hyphae by oxidative and nonoxidative killing mechanisms. Quantitative (i.e., neutropenia) or qualitative (i.e., associated with glucocorticoids, hyperglycemia, and/or acidosis) defects in phagocytic cell activity permit unrestricted growth of the hyphal form and invasive infection. Iron metabolism has a central role in pathogenesis of mucormycosis [6-8]. Thus, patients with iron overload states, including individuals undergoing chelation therapy with deferoxamine, are uniquely predisposed to mucormycosis. Of interest, deferoxamine acts as a siderophore for Mucorales species and promotes in vitro fungal growth. Similarly, the increased availability of serum iron in patients 
with diabetic acidosis partially accounts for their unique susceptibility to mucormycosis. As opposite to deferoxamine, other iron chelators such as deferasirox lack xenosiderophore activity for Rhizopus induce an iron-starvation effect to the fungus and have shown to be protective in animal models of mucormycosis [6-8].

Although Mucorales have not be reported to be entomopathogenic, we recently found that as opposite to other medically important filamentous fungi, injection of different Mucorales species in wild-type D. melanogaster results in a hyperacute infection, with disseminated fungal proliferation and high mortality rates [23]. Several aspects of immunopathogenesis of mucormycosis in humans were modeled in Drosophila, including increased host susceptibility following administration of corticosteroids, and the iron chelator deferoxamine. Of interest, the use of another iron chelator, deferasirox, which induces iron starvation to Mucorales spp and protects mice and possibly humans from infection, also significantly protected Drosophila from mucormycosis. In addition, Cunninghamella berthollethiae, which appears to be the most virulent Mucorales species in humans, exhibited increased virulence in comparison to other Mucorales species in the fly model [23].

The fly model of mucormycosis has been established in wild-type Drosophila, which obviates the need for crossings and allows for simple and rapid assessment of research questions in Mucorales pathogenicity. Thus, flies were recently implemented to evaluate the role of endosymbiotic toxinproducing bacteria in the virulence of Rhizopus species. Although a significant number of clinical Rhizopus isolates were found to harbor rhizoxin-producing bacteria, there was no difference in fungal virulence following antibiotic mediated eradication of the endosimbionts in both Drosophila and mice [48]. In addition, the association of increased voriconazole use with the emergence of Mucorales infection in immunocompromised patients was recently tested in the fly model. Surprisingly, preexposure of Mucorales to this newer triazole dramatically increased susceptibility of fruit flies to mucormycosis in Toll-independent fashion, which was also observed in the mouse model [49]. Collectively, these studies demonstrate that Mucorales species have developed common virulence strategies to invade evolutionarily disparate organisms such as Drosophila and humans.

Of interest, virulence of Cunninghamella in the fly model is significantly affected by the composition of fungal culture media, possibly reflecting differences in acquisition of iron or other nutritional factors [50]. In addition, because innate immunity in Drosophila is under circadian regulation, the timing of infection has significant impact in host defense against various pathogens, including filamentous fungi. In fact, genes involved in circadian rhythm regulation were significantly induced following infection with Mucorales species in Drosophila [23]. Furthermore, starvation of flies prior to infection confers protection against bacterial infection via release of $\mathrm{NO}$ [51], and possibly via regulating other immune-related pathways, such as FOXO signaling [40] and the autophagy response. Therefore, all these parameters need to be considered in virulence testing of Mucorales and other filamentous fungi in Drosophila.
Gene expression profiling in human monocytes and in immunocompromised mice infected with Rhizopus versus Aspergillus demonstrates a differential induction of immunerelated genes during mucormycosis [52], which likely reflects unique virulence traits of Mucorales species. Similarly, transcriptional profiling at early time points of infection in wildtype fruit flies infected with Rhizopus (pathogenic) versus Aspergillus (nonpathogenic) indicated distinct sets of genes that were selectively regulated in response to mucormycosis [23]. These genes could represent molecular targets for drug development aiming at modulating host immune response during infection. Of interest, a similar transcriptome profiling in Drosophila infected with two strains of Pseudomonas with different pathogenic properties revealed common groups of genes with those identified during $R h i-$ zopus infection of flies [53]. Notably, a group of genes down regulated following infection with the pathogenic strain in both studies included a skeletal muscle gene regulatory network under the control of cJun-N-terminal Kinase (JNK) pathway. Notably, activation of this pathway promoted local resistance to $P$. aeruginosa in flies and mice [54].

4.3. Other Emerging Filamentous Fungi. Fusarium and Scedosporium species are ubiquitous, saprophytic molds that are notoriously resistant to conventional antifungal agents [2]. These fungi have been increasingly reported causes of invasive, frequently fatal infections in immunosuppressed hosts. Occasionally, these opportunistic pathogens can cause difficult-to-treat localized infections in immunocompetent individuals with certain predisposing conditions, including onychomycosis, fungal keratitis, skin and soft tissue infection, and rarely brain abscesses [2]. Furthermore, as opposite to other filamentous fungi, Fusarium species have a unique predisposition for development of fungemia and disseminated necrotic skin lesions in severely immunocompromised patients [2]. These features suggest the existence of uncharacterized, unique virulence factors of these organisms. Drosophila melanogaster wild-type flies were recently found to be resistant to infection by different clinical isolates of Scedosporium, whereas Toll-deficient flies were highly susceptible to these fungi [24]. Of interest, Fusarium species caused lethal infection in wild-type flies although in a less acute mode of infection than in Toll deficient flies, an observation consistent with the ability of these fungi to infect a broad range of phylogenetically disparate hosts, ranging from plants to mammals. Although the lack of genetic tools currently precludes comprehensive analysis of virulence factors in these fungi, comparative analysis of host defense mechanisms during infection with these and other filamentous fungi in the Drosophila model could provide valuable information on the pathogenesis of these emerging infections.

\section{Antifungal Drug Efficacy Studies in Drosophila Models of Filamentous Fungi}

Drosophila has proven to be a reliable model for testing orally absorbed compounds with antifungal activity. In particular, voriconazole conferred significant protection in 
Toll-mutant flies infected with A. fumigatus [20]. Furthermore, the combination of voriconazole and terbinafine, two drugs that block sequential steps in the ergosterol pathway and show synergy in vitro against Aspergillus, was synergistic in the Drosophila model of aspergillosis [20]. Similarly, voriconazole preexposure was protective in flies infected with Fusarium moniliforme and S. apiospermum, but not in flies infected with $S$. prolificans, a finding that is consistent with in vitro susceptibilities of these species and in vivo studies in mice [24]. Besides conventional antifungal agents, administration of deferasirox, an iron chelator that induces iron starvation and exerts selective antifungal activity against Mucorales, significantly increased survival of flies in Drosophila model of mucormycosis [23].

Nonetheless, there are important limitations in the use of Drosophila and other invertebrate models in drug efficacy studies. Thus, precise estimation of the dose of a pharmacologic compound that is orally administered in flies is challenging. A more accurate way of drug delivery can be achieved by microinjection; however, this method is time consuming and requires technical training and specialized equipment in fruit flies. In addition, measurement of drug levels for pharmacokinetic analysis in Drosophila requires HPLC or bioassay methods that are more cumbersome, imprecise, and technically demanding in this model than in mammals [11]. For all these reasons, pharmacodynamic studies, which typically require multiple dosing of antifungal agents for long periods of time, are not feasible in Drosophila. Finally, the metabolism and elimination pathways of drugs and the potential for drug-drug interactions are largely unknown in Drosophila for most existing compounds.

Despite their limitations, Drosophila and other invertebrates are attractive models for mass-screening candidate antifungal compounds that will require subsequent validation in mammalian systems [55]. Such approaches have been used successfully in Drosophila to select life-extending compounds [56] and recently in C. elegans to identify compounds with novel mechanism of antifungal activity against Candida [57]. In the C. elegans study, thousands of synthetic and natural molecules were screened in a 96well plate liquid culture system and several compounds that exhibited in vivo activity without significant in vitro effect were selected, proving the benefits of this strategy. Notably, two of the 15 selected compounds identified in this screening exhibited potent antifungal activity in the mouse model of invasive candidiasis [57]. Overall, the simplicity, low cost, small size, and short generation time of invertebrate hosts make them ideal for high-throughput screening. As a proof of principle, many pharmaceutical and biotechnology companies are increasingly using minihost models for drug discovery. For example, Exelixis, Inc. (South San Francisco, CA) has created an extensive collection of Drosophila gene disruption strains for use in drug-target identification. Similarly, larger pharmaceutical companies such as Novartis (Basel, Switzerland) have created Drosophila functional genomics departments dedicated to the study of disease-related pathways and discovery of novel drug targets. Nonetheless, D. melanogaster models of infectious diseases are less amenable to automated mass screening for antimicrobial agents than are C. elegans models because of technical limitations associated with the size of the animals, methods of infection, frequent need for fly crosses to generate the desired mutants, and inability of adult flies to propagate in liquid culture systems.

\section{Implementing RNAi Screens to Identify Host and Pathogen Determinants of Immunopathogenesis of Fungal Diseases}

Over the past few years, Drosophila melanogaster S2 cells and RNAi technology have been successfully implemented to identify host factors implicated in pathogenesis of infections caused by intracellular pathogens $[29,30,58]$. There are many features of the Drosophila cell system that make it an attractive tool for these studies. Hence, the fly genome is highly annotated and fundamental innate immune pathways are evolutionarily conserved in Drosophila S2 macrophagelike cells. Furthermore, gene silencing is easier to perform in a high-throughput basis in Drosophila cell lines when compared to mammalian macrophage cell lines. Finally, Drosophila S2 cells have a successful track record in identifying novel host factors involved in phagocytosis and killing of many intracellular microbial pathogens, which have been subsequently validated in their mammalian cell counterparts $[29,30,58]$. In regard to fungal pathogens, investigators recently used an RNAi library of S2 cells to study genes involved in phagocytosis of C. albicans and identified novel genes encoding for proteins that specifically recognize and promote phagocytosis of Candida yeast cells [33]. Another RNAi screen in S2 cells was designed to select host factors that restrict intracellular survival and proliferation of the pathogenic fungus, Cryptococcus neoformans [36]. This study identified novel host genes implicated in Cryptococcus pathogenesis and revealed that proteins of the autophagy pathway are important for intracellular elimination of the fungus both in Drosophila S2 cells and mammalian macrophages.

In vitro, high-throughput screening strategies using phagocytic D. melanogaster cell lines have certain limitations. First, only host factors important for the intracellular life cycle of a pathogen can be tested. This approach is well suited for intracellular pathogens but not for extracellular organisms such as filamentous fungi. Thus, in contrast with bacteria, fungi have distinct replication stages (e.g., spore to hyphal transition) and relatively slow growth rates, which make difficult the establishment of reliable highthroughput phagocytosis and/or killing in vitro assays. In addition, silencing of important innate immune-related pathways may be missed in an in vitro screen because it may result in nonviable phenotypes, which can only be assessed using tissue-specific silencing in vivo. Finally, the complexity and dynamics of in vivo host-pathogen interplay, including tissue-specific host immune responses, cannot be reliably evaluated using an in vitro culture system.

Studies using conditional RNAi in D. melanogaster to analyze gene function in real time and a tissue-specific manner could overcome limitations of in vitro large-scale screening. In fact, the in vivo RNAi library for Drosophila flies 


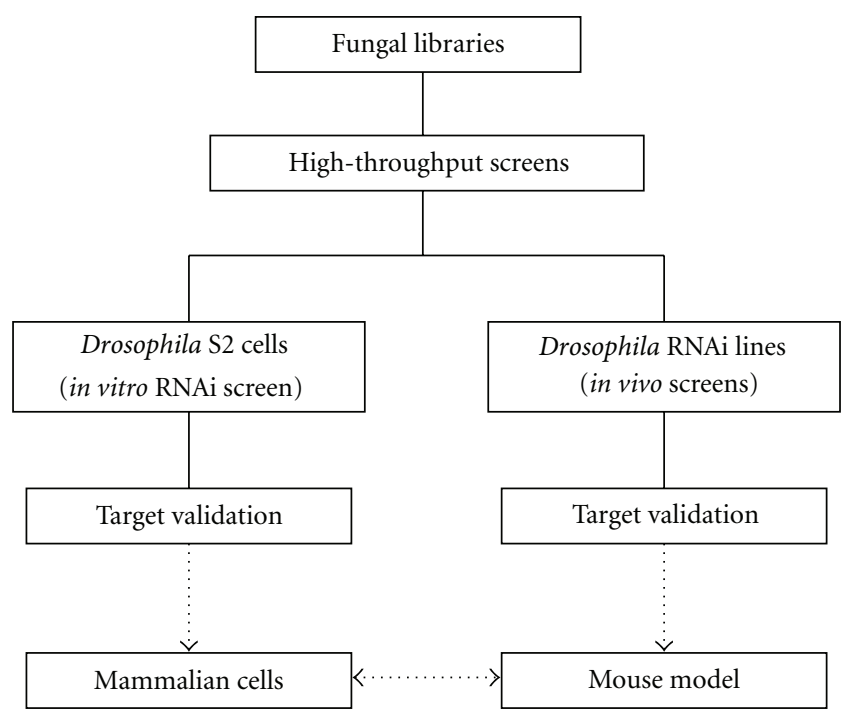

FIgURE 1: Prioritizing use of Drosophila model in selection of novel determinants of fungal pathogenicity in humans.

TABLE 1: Important research questions on antifungal immunity in Drosophila.

Which host defense mechanisms in Drosophila are important for fungal clearance before the induction of humoral immune responses? What is the contribution of cellular immunity in defense against fungi?

How Drosophila immune system discriminates opportunistic from entomopathogenic fungi?

Which fungal molecules other than b-glucans trigger activation of host immune responses in Drosophila?

Are there any fungal virulence factors that exert immunosuppressive effects on Toll signaling or other immune

signaling pathways?

Is there any role of pathogen or self-nucleic acid sensing in Drosophila host defense?

How Drosophila discriminates sensing of self- from non-self-immune activating molecules?

Is there any evidence of the presence of endogenous ligands for Drosophila pattern recognition receptors?

Are there any immune modulating properties of the antimicrobial peptides in Drosophila?

Is there any role for antimicrobial peptide-DNA complex formation in insect immunity against fungi?

Is there any cooperative activity between different Drosophila immune receptors?

has became commercially available [59], and a pilot genomewide in vivo screen in D. melanogaster designed to identify genes involved in epithelial host defense against an intestinal bacterial pathogen was recently completed [38]. For the first time, this study showed that the JAK-STAT signaling pathway has an important role in host defense against infections with bacterial pathogens in the gut by regulating epithelial cell homeostasis.

\section{Limitations of Drosophila Model of Fungal Infections}

Drosophila offers unique advantages in dissecting immunopathogenesis of fungal diseases because of its powerful genetics and highly conserved immune pathways. Nonetheless, the fly model also has some obvious limitations. For example, implementing Toll-deficient flies as model for virulence testing in a Mycology laboratory requires some degree of training for proper maintenance and crossing of Drosophila stocks, and basic equipment for manipulating, anesthetizing, and infecting the animals. Alternatively, use of larger in size invertebrates, such as Galleria mellonella, which are easier to infect and allow for infection at mammalian temperatures, could overcome some technical difficulties of the Drosophila model [60]. However, because in Galleria mellonella genetic tools are not available and innate immune pathways are less well characterized, this model is not suitable for in-depth analysis of host-related factors mediating fungal pathogenesis.

When compared to conventional animal models, the considerable difference in the anatomic structures of invertebrates and mammals raises questions on the pathophysiologic relevance of some $D$. melanogaster infection models. This may be particularly true for pathogens with life cycles adapted to mammalian hosts, or those that express their virulence mechanisms in a tissue-specific environment. For example, establishing a model of Pneumocystis jirovecii in 
invertebrate hosts [61] is not feasible. Nonetheless, even in mammalian hosts, some virulence attributes of pathogenicity may be dispensable to certain pathophysiologic settings or infection sites. For example, researchers recently showed that gliotoxin production was required for $A$. fumigatus pathogenicity in corticosteroid-immunosuppressed mice but not in neutropenic mice [62]. Furthermore, D. melanogaster lacks important constituents of human immunity, including a functional adaptive immune response, highly specialized innate immune cell subsets (e.g., dendritic cells, natural killer cells), and a complex network of cytokines, chemokines, and other effector molecules that have critical roles in orchestrating cell communication and regulation of inflammation and tolerance during infection. Overall, despite the considerable similarities in innate immune mechanisms, invertebrate models are not directly comparable with mammalian models. Thus, it is reasonable to speculate that some of the virulence attributes of Aspergillus and other filamentous fungi that affect mammals might not be important in invertebrate minihost models. Therefore, Drosophila must be viewed as a complementary, high-throughput genetic model, which could accelerate identification of novel host and pathogen determinants with a relevant role in development of fungal diseases in humans (Figure 1).

\section{Future Directions in Fungal Immunology Research in Drosophila}

The identification of the Drosophila melanogaster Toll signaling cascade and the subsequent characterization of mammalian Toll-like receptors (TLRs) have fundamentally altered our understanding of innate immunity. However, much remains to be learned on evolutionarily conserved antifungal immune defense mechanisms in Drosophila (Table 1). For example, whether immunostimulatory molecules of fungi other than b-glucans trigger immune recognition in Drosophila has not been elucidated. In addition, it is unknown whether and how Drosophila discriminates between pathogenic and opportunistic fungi. Similarly, the contribution of cellular immunity and Toll-independent mechanisms of antifungal host defense in fruit flies remains to be explored. Since the identification of mammalian TLRs, it became evident that nucleic acid sensing is an important aspect in pathogen recognition. Hence, dedicated endosomal TLRs and cytoplasmic pattern recognition receptors are specialized in sensing bacterial and viral nucleic acids and trigger robust inflammatory responses. Recent studies also demonstrate an important role for DNA neutrophil extracellular trap (NET) formation during bacterial and fungal infections [63]. In Drosophila, the role of nucleic acid sensing in immunity is largely unknown. Nonetheless, recent studies in other insects suggest that DNA NET formation is important for innate antibacterial immunity [64]. Finally, in humans, evolutionarily conserved antimicrobial peptides exert important immunomodulatory properties besides their direct effector function, by acting on various chemokine and signaling receptors $[65,66]$. Therefore, whether Drosophila antimicrobial peptides retain a similar role is an important research direction in understanding the evolution of mammalian immune system.

\section{Conflict of Interest}

D. P. Kontoyiannis has received research support and honoraria from Schering-Plough, Pfizer, Astella Pharma US, Inc., Enzon Pharmaceuticals, and Merck \& Co., Inc. G. Samonis has received honoraria from Schering-Plough, Astella Pharma US, Inc., Enzon Pharmaceuticals, and Merck and Co., Inc. G. Hamilos and G. Samonis have no conflicts of interest.

\section{Acknowledgments}

The authors thank G. Halder for valuable contributions in establishing Drosophila models of fungal infection, Nathaniel Albert for excellent technical assistance, and all current and past members of the Kontoyiannis Laboratory for useful discussions and contributions. This work was supported in part by The University of Texas MD Anderson Cancer Center E. N. Cobb Faculty Scholar Endowment and the National Institutes of Health through MD Anderson's Cancer Center Support Grant CA016672 (to D. P. Kontoyiannis) and the Marie Curie International Reintegration Grant IRG-260210 (to G. Chamilos).

\section{References}

[1] S. K. Fridkin, "The changing face of fungal infections in health care settings," Clinical Infectious Diseases, vol. 41, no. 10, pp. 1455-1460, 2005.

[2] M. Nucci and K. A. Marr, "Emerging fungal diseases," Clinical Infectious Diseases, vol. 41, no. 4, pp. 521-526, 2005.

[3] K. A. Marr, R. A. Carter, F. Crippa, A. Wald, and L. Corey, "Epidemiology and outcome of mould infections in hematopoietic stem cell transplant recipients," Clinical Infectious Diseases, vol. 34, no. 7, pp. 909-917, 2002.

[4] J. P. Latgé, “The pathobiology of Aspergillus fumigatus," Trends in Microbiology, vol. 9, no. 8, pp. 382-389, 2001.

[5] T. R. T. Dagenais and N. P. Keller, "Pathogenesis of Aspergillus fumigatus in invasive aspergillosis," Clinical Microbiology Reviews, vol. 22, no. 3, pp. 447-465, 2009.

[6] J. A. Ribes, C. L. Vanover-Sams, and D. J. Baker, "Zygomycetes in human disease," Clinical Microbiology Reviews, vol. 13, no. 2, pp. 236-301, 2000.

[7] B. Spellberg, J. Edwards, and A. Ibrahim, "Novel perspectives on mucormycosis: pathophysiology, presentation, and management," Clinical Microbiology Reviews, vol. 18, no. 3, pp. 556-569, 2005.

[8] D. P. Kontoyiannis and R. E. Lewis, "Invasive zygomycosis: update on pathogenesis, clinical manifestations, and management," Infectious Disease Clinics of North America, vol. 20, no. 3, pp. 581-607, 2006.

[9] F. Tekaia and J. P. Latgé, “Aspergillus fumigatus: saprophyte or pathogen?" Current Opinion in Microbiology, vol. 8, no. 4, pp. 385-392, 2005.

[10] L. Romani, "Immunity to fungal infections," Nature Reviews Immunology, vol. 11, pp. 275-288, 2011.

[11] K. V. Clemons and D. A. Stevens, "The contribution of animal models of aspergillosis to understanding pathogenesis, 
therapy and virulence," Medical Mycology, vol. 43, no. 1, pp. S101-S110, 2005.

[12] E. Pradel and J. J. Ewbank, "Genetic models in pathogenesis," Annual Review of Genetics, vol. 38, pp. 347-363, 2004.

[13] B. Lemaitre, E. Nicolas, L. Michaut, J. M. Reichhart, and J. A. Hoffmann, "The dorsoventral regulatory gene cassette spatzle/Toll/Cactus controls the potent antifungal response in Drosophila adults," Cell, vol. 86, no. 6, pp. 973-983, 1996.

[14] E. Mylonakis, F. M. Ausubel, J. R. Perfect, J. Heitman, and S. B. Calderwood, "Killing of Caenorhabditis elegans by Cryptococcus neoformans as a model of yeast pathogenesis," Proceedings of the National Academy of Sciences of the United States of America, vol. 99, no. 24, pp. 15675-15680, 2002.

[15] Y. Apidianakis, L. G. Rahme, J. Heitman, F. M. Ausubel, S. B. Calderwood, and E. Mylonakis, "Challenge of Drosophila melanogaster with Cryptococcus neoformans and role of the innate immune response," Eukaryotic Cell, vol. 3, no. 2, pp. 413-419, 2004.

[16] M. S. Lionakis, R. E. Lewis, G. S. May et al., "Toll-deficient Drosophila flies as a fast, high-throughput model for the study of antifungal drug efficacy against invasive aspergillosis and Aspergillus virulence," Journal of Infectious Diseases, vol. 191, no. 7, pp. 1188-1195, 2005.

[17] A. M. Alarco, A. Marcil, J. Chen, B. Suter, D. Thomas, and M. Whiteway, "Immune-deficient Drosophila melanogaster: a model for the innate immune response to human fungal pathogens," Journal of Immunology, vol. 172, no. 9, pp. 5622$5628,2004$.

[18] G. Chamilos, M. S. Lionakis, R. E. Lewis et al., "Drosophila melanogaster as a facile model for large-scale studies of virulence mechanisms and antifungal drug efficacy in Candida species," Journal of Infectious Diseases, vol. 193, no. 7, pp. 1014-1022, 2006.

[19] G. Chamilos, R. E. Lewis, J. Hu et al., "Drosophila melanogaster as a model host to dissect the immunopathogenesis of zygomycosis," Proceedings of the National Academy of Sciences of the United States of America, vol. 105, no. 27, pp. 9367-9372, 2008.

[20] G. A. Lamaris, G. Chamilos, R. E. Lewis, and D. P. Kontoyiannis, "Virulence studies of Scedosporium and Fusarium species in Drosophila melanogaster," Journal of Infectious Diseases, vol. 196, no. 12, pp. 1860-1864, 2007.

[21] G. Chamilos, M. S. Lionakis, R. E. Lewis, and D. P. Kontoyiannis, "Role of mini-host models in the study of medically important fungi," Lancet Infectious Diseases, vol. 7, no. 1, pp. 42-55, 2007.

[22] N. Vodovar, C. Acosta, B. Lemaitre, and F. Boccard, "Drosophila: a polyvalent model to decipher host-pathogen interactions," Trends in Microbiology, vol. 12, no. 5, pp. 235242, 2004.

[23] E. Mylonakis and A. Aballay, "Worms and flies as genetically tractable animal models to study host-pathogen interactions," Infection and Immunity, vol. 73, no. 7, pp. 3833-3841, 2005.

[24] K. Kavanagh and E. P. Reeves, "Exploiting the potential of insects for in vivo pathogenicity testing of microbial pathogens," FEMS Microbiology Reviews, vol. 28, no. 1, pp. 101-112, 2004.

[25] D. Ferrandon, J. L. Imler, C. Hetru, and J. A. Hoffmann, "The Drosophila systemic immune response: sensing and signalling during bacterial and fungal infections," Nature Reviews Immunology, vol. 7, no. 11, pp. 862-874, 2007.

[26] A. A. Matskevich, J. Quintin, and D. Ferrandon, "The Drosophila PRR GNBP3 assembles effector complexes involved in antifungal defenses independently of its Toll- pathway activation function," European Journal of Immunology, vol. 40, no. 5, pp. 1244-1254, 2010.

[27] M. Gottar, V. Gobert, A. A. Matskevich et al., "Dual detection of fungal infections in Drosophila via recognition of glucans and sensing of virulence factors," Cell, vol. 127, no. 7, pp. 1425-1437, 2006.

[28] E. R. Haine, Y. Moret, M. T. Siva-Jothy, and J. Rolff, "Antimicrobial defense and persistent infection in insects," Science, vol. 322, no. 5905, pp. 1257-1259, 2008.

[29] H. Agaisse, L. S. Burrack, J. A. Philips, E. J. Rubin, N. Perrimon, and D. E. Higgins, "Genome-wide RNAi screen for host factors required for intracellular bacterial infection," Science, vol. 309, no. 5738, pp. 1248-1251, 2005.

[30] J. A. Philips, E. J. Rubin, and N. Perrimon, "Drosophila RNAi screen reveals CD36 family member required for mycobacterial infection," Science, vol. 309, no. 5738, pp. 12511253, 2005.

[31] C. Kocks, J. H. Cho, N. Nehme et al., "Eater, a transmembrane protein mediating phagocytosis of bacterial pathogens in Drosophila," Cell, vol. 123, no. 2, pp. 335-346, 2005.

[32] S. Blandin, S. H. Shiao, L. F. Moita et al., "Complement-like protein TEP1 is a determinant of vectorial capacity in the malaria vector Anopheles gambiae," Cell, vol. 116, no. 5, pp. 661-670, 2004.

[33] S. L. Stroschein-Stevenson, E. Foley, P. H. O'Farrell, and A. D. Johnson, "Identification of Drosophila gene products required for phagocytosis of Candida albicans," PLoS Biology, vol. 4, no. 1, article e4, pp. 0087-0099, 2006.

[34] A. Levitin, A. Marcil, G. Tettweiler et al., "Drosophila melanogaster Thor and response to Candida albicans infection," Eukaryotic Cell, vol. 6, no. 4, pp. 658-663, 2007.

[35] E. Foley and P. H. O'Farrell, "Nitric oxide contributes to induction of innate immune responses to gram-negative bacteria in Drosophila," Genes and Development, vol. 17, no. 1, pp. 115-125, 2003.

[36] Q.-M. Qin, J. Luo, X. Lin et al., "Functional analysis of host factors that mediate the intracellular lifestyle of cryptococcus neoformans," PLoS Pathogens, vol. 7, no. 6, article e1002078, 2011.

[37] E. M. Ha, C. T. Oh, Y. S. Bae, and W. J. Lee, "A direct role for dual oxidase in Drosophila gut immunity," Science, vol. 310, no. 5749, pp. 847-850, 2005.

[38] S. J. F. Cronin, N. T. Nehme, S. Limmer et al., "Genome-wide RNAi screen identifies genes involved in intestinal pathogenic bacterial infection," Science, vol. 325, no. 5938, pp. 340-343, 2009.

[39] M. T. Glittenberg, I. Kounatidis, D. Christensen et al., "Pathogen and host factors are needed to provoke a systemic host response to gastrointestinal infection of Drosophila larvae by Candida albicans," DMM Disease Models and Mechanisms, vol. 4, no. 4, pp. 515-525, 2011.

[40] T. Becker, G. Loch, M. Beyer et al., "FOXO-dependent regulation of innate immune homeostasis," Nature, vol. 463, no. 7279, pp. 369-373, 2010.

[41] J. Chen, C. Xie, L. Tian, L. Hong, X. Wu, and J. Han, "Participation of the p38 pathway in Drosophila host defense against pathogenic bacteria and fungi," Proceedings of the National Academy of Sciences of the United States of America, vol. 107, no. 48, pp. 20774-20779, 2010.

[42] M. T. Glittenberg, S. Silas, D. M. MacCallum, N. A.R. Gow, and P. Ligoxygakis, "Wild-type Drosophila melanogaster as an alternative model system for investigating the pathogenicity of Candida albicans," DMM Disease Models and Mechanisms, vol. 4, no. 4, pp. 504-514, 2011. 
[43] G. Chamilos, E. M. Bignell, M. Schrettl et al., "Exploring the concordance of Aspergillus fumigatus pathogenicity in mice and Toll-deficient flies," Medical Mycology, vol. 48, no. 3, pp. 506-510, 2010.

[44] R. Bhabhra, M. D. Miley, E. Mylonakis et al., "Disruption of the Aspergillus fumigatus gene encoding nucleolar protein CgrA impairs thermotolerant growth and reduces virulence," Infection and Immunity, vol. 72, no. 8, pp. 4731-4740, 2004.

[45] R. Ben-Ami, G. A. Lamaris, R. E. Lewis, and D. P. Kontoyiannis, "Interstrain variability in the virulence of Aspergillus fumigatus and Aspergillus terreus in a Toll-deficient Drosophila fly model of invasive aspergillosis," Medical Mycology, vol. 48, no. 2, pp. 310-317, 2010.

[46] W. C. Nierman, A. Pain, M. J. Anderson et al., "Genomic sequence of the pathogenic and allergenic filamentous fungus Aspergillus fumigatus," Nature, vol. 438, no. 7071, pp. 11511156, 2006.

[47] G. Chamilos, C. J. Nobile, V. M. Bruno, R. E. Lewis, A. P. Mitchell, and D. P. Kontoyiannis, "Candida albicans Cas5, a regulator of cell wall integrity, is required for virulence in murine and toll mutant fly models," Journal of Infectious Diseases, vol. 200, no. 1, pp. 152-157, 2009.

[48] A. S. Ibrahim, T. Gebremariam, M. Liu et al., "Bacterial endosymbiosis is widely present among zygomycetes but does not contribute to the pathogenesis of mucormycosis," Journal of Infectious Diseases, vol. 198, no. 7, pp. 1083-1090, 2008.

[49] G. A. Lamaris, R. Ben-Ami, R. E. Lewis, G. Chamilos, G. Samonis, and D. P. Kontoyiannis, "Increased virulence of zygomycetes organisms following exposure to voriconazole: a study involving fly and murine models of zygomycosis," Journal of Infectious Diseases, vol. 199, no. 9, pp. 1399-1406, 2009.

[50] G. N. Pongas, R. Ben-Ami, R. E. Lewis, T. J. Walsh, and D. P. Kontoyiannis, "Culture medium composition affects the lethality of Cunninghamella bertholletiae in a fly model of mucormycosis," Antimicrobial Agents and Chemotherapy, vol. 53, no. 10, p. 4569, 2009.

[51] A. E. Brown, J. Baumbach, P. E. Cook, and P. Ligoxygakis, "Short-term starvation of immune deficient Drosophila improves survival to gram-negative bacterial infections," PLoS ONE, vol. 4, no. 2, Article ID e4490, 2009.

[52] D. P. Kontoyiannis, "Manipulation of host angioneogenesis: a critical link for understanding the pathogenesis of invasive mold infections?" Virulence, vol. 1, no. 3, pp. 192-196, 2010.

[53] Y. Apidianakis, M. N. Mindrinos, W. Xiao et al., "Profiling early infection responses: Pseudomonas aeruginosa eludes host defenses by suppressing antimocrobial peptide gene expression," Proceedings of the National Academy of Sciences of the United States of America, vol. 102, no. 7, pp. 2573-2578, 2005.

[54] Y. Apidianakis, M. N. Mindrinos, W. Xiao et al., "Involvement of skeletal muscle gene regulatory network in susceptibility to wound infection following trauma," PLoS ONE, vol. 2, no. 12, Article ID e1356, 2007.

[55] T. I. Moy, A. R. Ball, Z. Anklesaria, G. Casadei, K. Lewis, and F. M. Ausubel, "Identification of novel antimicrobials using a live-animal infection model," Proceedings of the National Academy of Sciences of the United States of America, vol. 103, no. 27, pp. 10414-10419, 2006.

[56] J. H. Bauer, S. Goupil, G. B. Garber, and S. L. Helfand, "An accelerated assay for the identification of lifespan-extending interventions in Drosophila melanogaster," Proceedings of the National Academy of Sciences of the United States of America, vol. 101, no. 35, pp. 12980-12985, 2004.
[57] J. Breger, B. B. Fuchs, G. Aperis, T. I. Moy, F. M. Ausubel, and E. Mylonakis, "Antifungal chemical compounds identified using a C. elegans pathogenicity assay.", PLoS Pathogens, vol. 3, no. 2, p. e18, 2007.

[58] L. W. Cheng, J. P. M. Viala, N. Stuurman, U. Wiedemann, R. D. Vale, and D. A. Portnoy, "Use of RNA interference in Drosophila S2 cells to identify host pathways controlling compartmentalization of an intracellular pathogen," Proceedings of the National Academy of Sciences of the United States of America, vol. 102, no. 38, pp. 13646-13651, 2005.

[59] G. Dietzl, D. Chen, F. Schnorrer et al., "A genome-wide transgenic RNAi library for conditional gene inactivation in Drosophila," Nature, vol. 448, no. 7150, pp. 151-156, 2007.

[60] J. L. Slater, L. Gregson, D. W. Denning, and P. A. Warn, "Pathogenicity of Aspergillus fumigatus mutants assessed in Galleria mellonella matches that in mice," Medical Mycology, vol. 49, supplement 1, pp. S107-S113, 2011.

[61] S. E. Evans, K. Leventakos, R. Ben-Ami et al., "Toll-deficient Drosophila are resistant to infection by Pneumocystis spp. additional evidence of specificity to mammalian hosts," Virulence, vol. 1, no. 6, pp. 523-525, 2010.

[62] S. Spikes, R. Xu, C. K. Nguyen et al., "Gliotoxin production in Aspergillus fumigatus contributes to host-specific differences in virulence," Journal of Infectious Diseases, vol. 197, no. 3, pp. 479-486, 2008.

[63] V. Brinkmann, U. Reichard, C. Goosmann et al., "Neutrophil extracellular traps kill bacteria," Science, vol. 303, no. 5663, pp. 1532-1535, 2004.

[64] B. Altincicek, S. Stötzel, M. Wygrecka, K. T. Preissner, and A. Vilcinskas, "Host-derived extracellular nucleic acids enhance innate immune responses, induce coagulation, and prolong survival upon infection in insects," Journal of Immunology, vol. 181, no. 4, pp. 2705-2712, 2008.

[65] R. Lande, J. Gregorio, V. Facchinetti et al., "Plasmacytoid dendritic cells sense self-DNA coupled with antimicrobial peptide," Nature, vol. 449, no. 7162, pp. 564-569, 2007.

[66] D. Ganguly, G. Chamilos, R. Lande et al., "Self-RNAantimicrobial peptide complexes activate human dendritic cells through TLR7 and TLR8," Journal of Experimental Medicine, vol. 206, no. 9, pp. 1983-1994, 2009. 

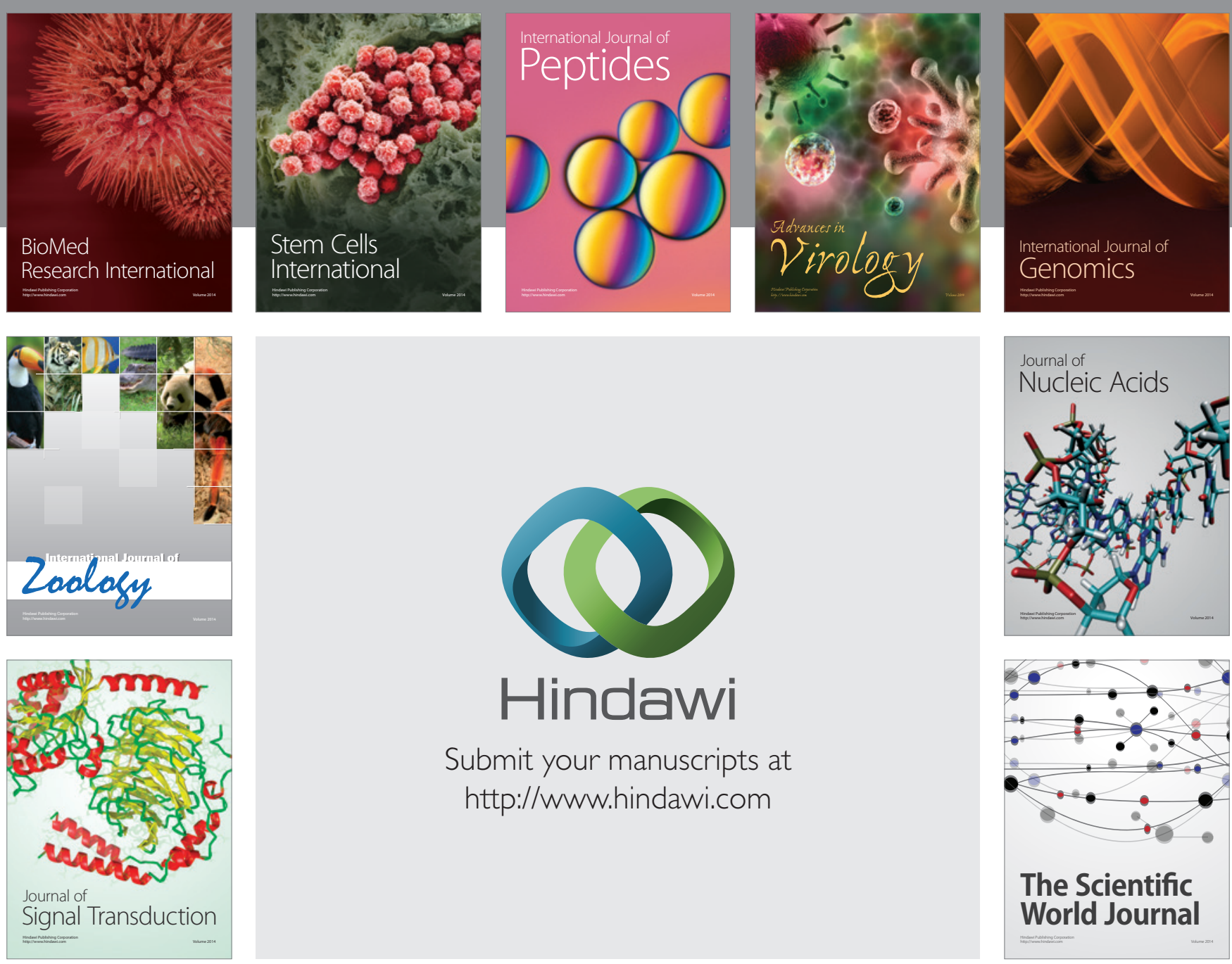

Submit your manuscripts at

http://www.hindawi.com
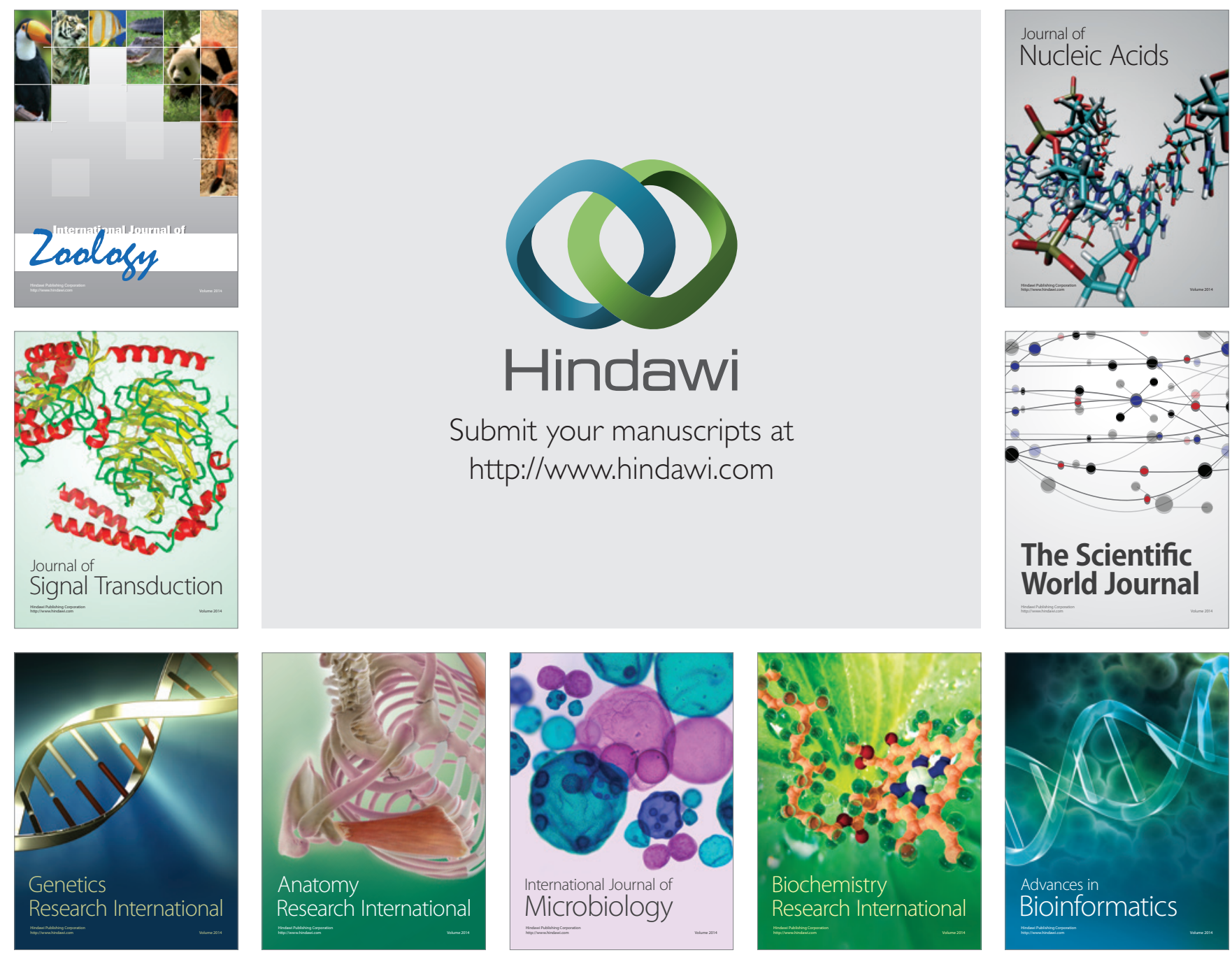

The Scientific World Journal
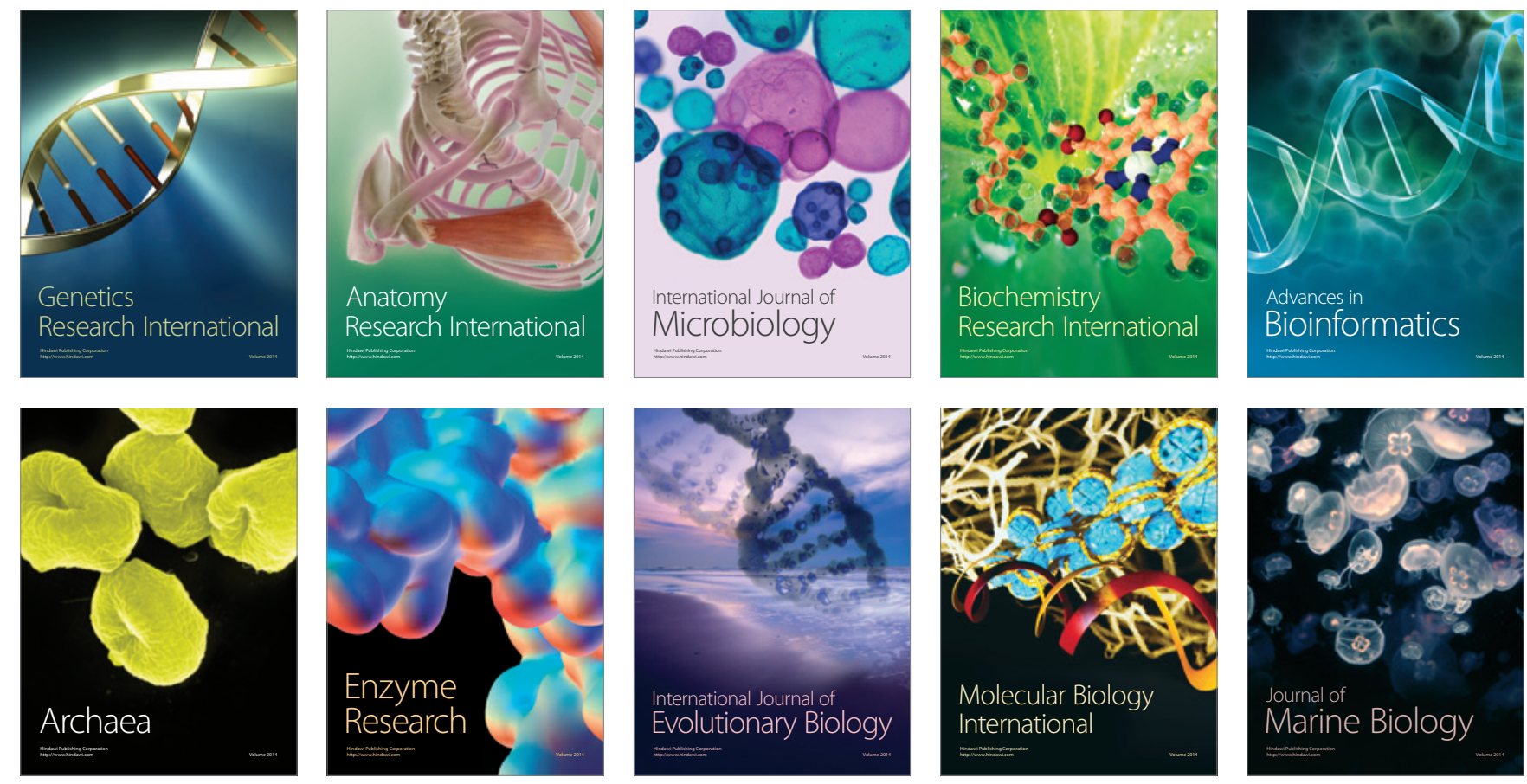